\title{
Idiopathic hypertrophic pachymeningitis with intracranial hypotension and distension of anterior internal vertebral venous plexus are associated with positional headaches but not sufficient in the diagnosis ${ }^{*}$
}

\author{
Yongpeng Yu ${ }^{1 \#}$, Changkai Sun ${ }^{2 \#}$, Chen Zhang ${ }^{3}$, Hongqin Zhao ${ }^{3}$, Thian C. $\mathrm{Ng}^{4}$ \\ ${ }^{1}$ Department of Neurology, The Affiliated Wendeng Center Hospital of Weifang Medical College and Teaching Hospital of Dalian \\ Medical University, Wendeng, China \\ ${ }^{2}$ Liaoning Provincial Key Discipline of Physiology, Institute of Brain Disorders and The Provincial Key Lab for Brain Disorders of \\ Liaoning Province, Dalian Medical University, Dalian, China \\ ${ }^{3}$ Department of Neurology, The Affiliated Hospital of Qingdao University Medical College, Qingdao, China \\ ${ }^{4}$ Radiology Department, National University of Singapore, Singapore City, Singapore \\ Email: \#yypeng6688@126.com
}

Received 21 April 2013; revised 28 May 2013; accepted 6 July 2013

Copyright (C) 2013 Yongpeng Yu et al. This is an open access article distributed under the Creative Commons Attribution License, which permits unrestricted use, distribution, and reproduction in any medium, provided the original work is properly cited.

\begin{abstract}
Idiopathic hypertrophic pachymeningitis (IHP) is a very infrequent disorder that causes a localized or diffuse thickening of the dura mater. The main clinical features of IHP at presentation include headache, cranial nerve involvement, ataxia, transient ischemic attacks (TIA) and seizures. Intracranial hypotension (ICH) and distension of anterior internal vertebral venous plexus (IVVP) have not previously been reported as the main clinical manifestations. We report a unique case with these characteristics as the main clinical findings, and investigate the internal relationship between these characteristics. This study highlights the heterogeneity of IHP, in terms of clinical, imaging, and pathological features. We recommend that dural biopsies should be considered in patients with clinically typical orthostatic headache and (or) diffuse pachymeningeal enhancement on magnetic resonance imaging (MRI) for the diagnosis of IHP.
\end{abstract}

Keywords: Idiopathic; Pachymeningitis; Intracranial Hypotension

\footnotetext{
*Informed consent was obtained from this patient. No potential conflict of interest relevant to this article was reported.

This study was supported by the National Science and Technology Major Project of China, (No. 2012ZX09503001) and the Technology Development Project of Weihai (No. 2011-2-91-2).

"Corresponding authors.
}

\section{INTRODUCTION}

Idiopathic hypertrophic pachymeningitis (IHP) is a rare idiopathic fibrosing inflammatory disease, characterized by marked diffuse thickening of the dura mater, causing progressive neurological deficits, such as headache, cranial neuropathies, cerebellar dysfunction and visual loss occurring either alone or in combination [1]. Intracranial hypotension (ICH) and distension of anterior internal vertebral venous plexus (IVVP) in association with this entity is rare. Here, we report a case of IHP with these clinical characteristics.

\section{CASE REPORT}

A 40-year-old man presented with a history of headache and neck ache for two and half months. His symptoms were worsened on standing and walking, but were relieved on lying down. He had no significant prior medical history and no systemic features of any chronic illness. On admission, he was afebrile and had normal vital signs. Neurologic examination was negative for focal motor or sensory deficits. His complete blood count, renal and liver function tests, thyroidfunction tests, tuberculin test, echocardiogram, electrocardiogram, urine analysis and angiotensin-converting enzyme E (ACE) levels were all unremarkable. Tumor, autoimmune and venereal disease markers were also not elevated. Other laboratory values were as follows: $\mathrm{C}$ reactive protein (CRP), 16.6 $\mathrm{mg} / \mathrm{L}$; and erythrocyte sedimentation rate (ESR), $32 \mathrm{~mm} / \mathrm{h}$. A cerebrospinal fluid (CSF) examination revealed an 
opening pressure of $60 \mathrm{~mm} \mathrm{H}_{2} \mathrm{O}, 12$ cells/ $\mathrm{mm}^{3}$ (all mononuclear), protein of $0.49 \mathrm{~g} / \mathrm{L}$ and glucose of $2.35 \mathrm{mmol} / \mathrm{L}$ with corresponding blood glucose of $5.47 \mathrm{mmol} / \mathrm{L}$. CSF examination for syphilis, fungus, bacteria and malignant cells were all negative. His chest X-ray was normal. Sagittal T1-weighted magnetic resonance imaging (MRI) of the cervical and upper cervical spine showed a mass of isointense signal intensity. On T2-weighted MRI, a mass of slightly high signal intensity were seen diffusely in the dura within the spinal canal located adjacent to the posterior aspect of the C1 through C7 vertebral bodies. The corresponding sagittal contrast-enhanced T1-weighted MRI at the same level revealed thick enhancement of the anterior epidural mass with a central, non-enhancing area (flow-void) (Figure 1), which is regarded as IVVP. Sometimes symptoms and signs of a disease may occur out of the expected or textbook sequence. Fine needle aspiration cytology showed a dense fibrous cellular tissue (Figure 2) with no evidence of granulomas, vasculitis or any infective pathogens. The diagnosis of IHP with IVVP was confirmed. He was treated with intravenous methylprednisolone $(50 \mathrm{mg} / \mathrm{d})$ and intravenous rehydration therapy for 7 days, followed by oral prednisolone at $0.5 \mathrm{mg} / \mathrm{kg}$ per day with a plan to reduce it slowly over 2 weeks to 3 weeks. His symptoms improved significantly during his hospitalization stay and continued to be clinically asymptomatic for one year after his leaving hospital.
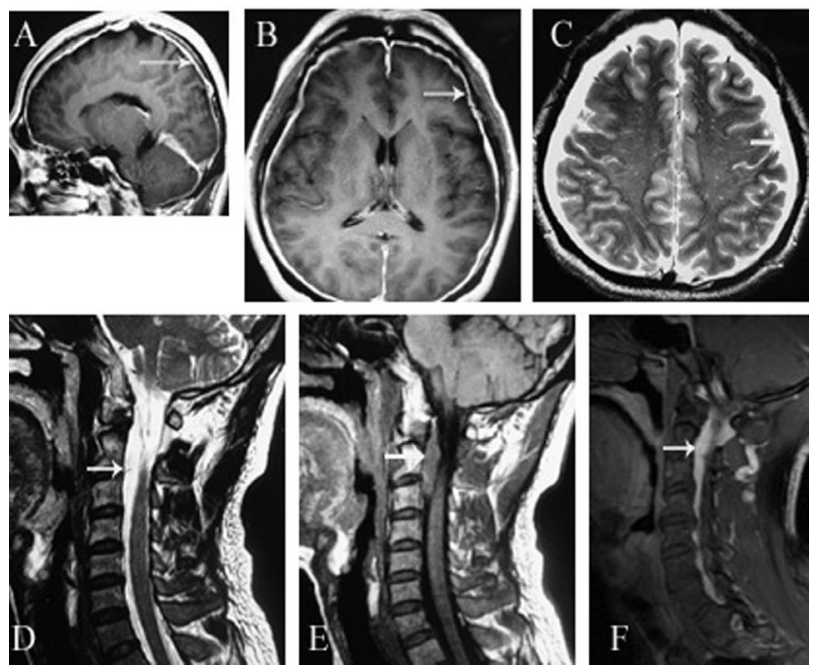

Figure 1. Axial (A) and axial (B) T1WI, T2WI and contrastenhanced MRI (C) show markedly thickened and enhanced dura extending from the vertex to the tentorium. (D) Sagittal T2WI of the cervical and upper thoracic spine shows a mass of very high signal intensity (arrows) and a mass of low signal intensity in T1WI (E), which is within the spinal canal located adjacent to the posterior aspect of the $\mathrm{C} 1$ through $\mathrm{T} 1$ vertebral bodies. (F) There is also thickening and hypointensity of the dura anterior to the spinal cord in T1WI (arrowheads).
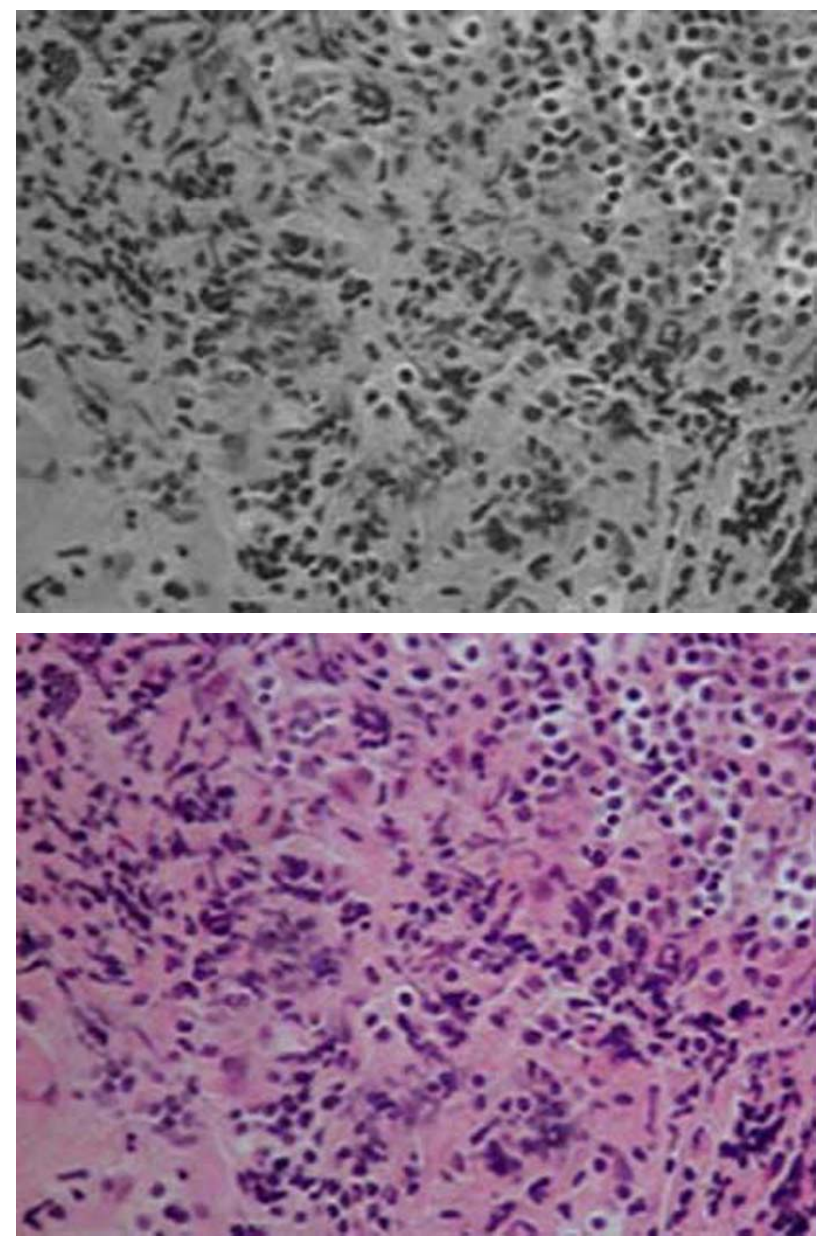

Figure 2. Histopathological examination shows that chronic inflammatory infiltrate is consisting chiefly of plasma cells with additional lymphocytes and scattered histiocytes $(\times 40)$.

\section{DISCUSSION}

IHP usually presents as a chronic progressive disease with manifestations such as headache, cranial neuropathies, cerebellar dysfunction, obstructive hydrocephalus, seizure, sinus thrombosis, radiculopathy, myelopathy and visual loss occurring either alone or in combination [2,3]. Neuroimaging is essential in the diagnostic work-up of suspected IHP, with the characteristic appearance of linear or nodular gadolinium enhancement typically involving the falx cerebri, tentorium and cavernous sinus. To our knowledge, the spinal form is extremely rare with only two cases described previously [4]. Based on the Monro-Kellie doctrine [5], we hypothesize that a decrease in CSF volume may lead to compensatory enlargement of both the cerebral and spinal venous plexuses. This distension of the anterior IVVP may have contributed to an inflammatory response of the dura. A previous study has proposed that the prominence of spinal venous dilatation may be an important sign for establishing the diagnosis of ICH by MRI [5]. This case is 
usually presented with the feature of diffuse pachymeningeal enhancement on MRI and the core or cardinal symptom of postural headache as well as the low CSF pressure. However, in our case, the dural biopsy confirmed the diagnosis of IHP.

\section{CONCLUSION}

ICH and IHP are so similar as to be easily confused. Therefore, to avoid clinical errors, application of neuroimaging in ICH should be correlated with the patient's biopsy results.

\section{REFERENCES}

[1] Prabhakar, S., Bhatia, R., Lal, V. and Singh, P. (2002) Hypertrophic pachymeningitis: Varied manifestations of a single disease entity. Neurology India, 50, 45-52.
[2] Kupersmith, M.J., Martin, V., Heller, G., et al. (2004) Idiopathic hypertrophic pachymeningitis. Neurology, 62, 686-694. doi:10.1212/01.WNL.0000113748.53023.B7

[3] Bhatia, R., Tripathi, M., Srivastava, A., et al. (2009) Idiopathic hypertrophic cranial pachymeningitis and dural sinus occlusion: Two patients with long-term follow up. Journal of Clinical Neuroscience, 16, 937-942. doi:10.1016/j.jocn.2008.08.012

[4] Pai, S., Welsh, C.T., Patel, S., et al. (2007) Idiopathic hypertrophic spinal pachymeningitis: Report of two cases with typical MR imaging findings. American Journal of Neuroradiology, 28, 590-592.

[5] Yousry, I., Förderreuther, S., Moriggl, B., et al. (2001) Cervical MR imaging in postural headache: MR signs and pathophysiological implications. American Journal of Neuroradiology, 22, 1239-1250. 